\title{
The Mark IV VLBI Data-Acquisition and Correlation System
}

\author{
ALAN R. WHITNEY \\ MIT Haystack Observatory \\ Rt. 40 \\ Westford, Massachusetts, USA 01886
}

\begin{abstract}
Modern VLBI observations for both astronomy and geodesy continue to demand the utmost in sensitivity. Of the methods potentially available for increasing the sensitivity of continuum VLBI observations, increasing the recorded bandwidth is generally the most cost effective.

Over the past two years a broadly-supported program has been underway at Haystack Observatory to increase the sensitivity of the Mark IIIA VLBI system by more than a factor of 2 . The result is an upgrade to the existing Mark IIIA data-acquisition system, dubbed Mark IV, which increases the maximum data rate to $1024 \mathrm{Mbits} / \mathrm{sec}$, more than quadrupling the maximum data-rate of the Mark IIIA..

A new correlator, based on a new custom VLSI correlator chip is also being designed to support the $1 \mathrm{Gbit} / \mathrm{sec}$ data rates from the Mark IV data-acquisition-system. An international collaborative effort is being mounted to help defray the high costs of development.
\end{abstract}

\section{Introduction}

The progress of geodetic VLBI over the last decade has been truly dramatic. Following is a partial list of a few of the many advances that have been made:

- Distances between radio telescopes distributed widely over the world are routinely measured to the order of $1 \mathrm{~cm}$, meeting and exceeding the original goals of the NASA Crustal Dynamics Project. As a result, the motion of tectonic plates, typically a few $\mathrm{cm} /$ year, has been measured directly for the first time, and a new goal of mm-level measurements has been set.

- Specially-instrumented and conducted R\&D experiments in the fall of 1989 showed measurement precision and repeatability of $\sim 3 \mathrm{~mm}$ on baselines thousands of kilometers in length.

- VLBI routinely provides the most precise measurements available of the earth's rotation rate and spin-axis orientation.

These advances have been largely fueled by a broad spectrum of technological advances. Among these are:

- Routine use of the Mark III system beginning in the early eighties increased the recording bandwidth of VLBI data from $4 \mathrm{Mbits} / \mathrm{sec}$ of the Mark II system to more than 200 MBits/sec, improving the basic sensitivity of continuum observations by more than a factor of 7 .

- Development of the Mark III and Mark IIIA correlators have allowed unprecedented volumes of VLBI data to be routinely processed. In the ten years from 1980, an estimated 3 million observations have been processed.

- Improved receiver systems with lower noise and wider-bandwidths 
- Improved phase-calibration systems and cable-calibration systems to remove small instrumental effects

- Improved data-analysis techniques and software

Though the advances both scientifically and technologically have been impressive, there is still much room and need for growth. The Mark IV VLBI system has grown out of this need. Conceived as an upgrade to the existing Mark IIIA system, and yet compatible with both Mark IIIA and VLBA systems, the Mark IV system pushes VLBI recording and processing technology to more than $1 \mathrm{Gbit} / \mathrm{sec} /$ station.

\section{Mark IV Data-Acquisition System}

In early 1990 a laboratory demonstration of a $1 \mathrm{Gbit} / \mathrm{sec}$ VLBI data-acquisition system was conducted by modifying a VLBA recording system ${ }^{1}$. The Mark IIIA recording system uses exactly the same Honeywell tape transport and headstack assembly as the VLBA recorder used in the 1-Gbit/sec demonstration; therefore, the Mark IIIA data recording system may be upgraded in a similar fashion to that demonstrated in the 1-Gbit/sec demonstration, provided the necessary formatter and write electronics are provided.

The standard Mark IIIA data-acquisition-system configuration provides 14 video converters, each with independent upper and lower-sideband channels of $4 \mathrm{MHz}$ bandwidth maximum each, for a total maximum bandwidth of $112 \mathrm{MHz}$. The existing formatter provides 28 channels of data at 1-bit/sample, at a maximum sample rate of $8 \mathrm{Mbits} / \mathrm{sec} / \mathrm{channel}$, for a total maximum data rate of $224 \mathrm{Mbits} / \mathrm{sec}$. Two identical standard high-density headstacks are mounted in the Mark IIIA recorder, one headstack dedicated to writing and the other to reading. Of the 36 tracks available in each headstack, only 28 are used by the Mark IIIA system, each track independently carrying data from a single video-converter sideband.

The following actions are necessary to increase the maximum data rate of the Mark IIIA to $1024 \mathrm{Mbits} / \mathrm{sec}$ :

- Increase the number of video-converters from 14 to 16 ; this will be done by utilizing an existing rack-mounted spare and adding one more video-converter to the rack.

- Increase the maximum bandwidth of a video-converter sideband to $8 \mathrm{MHz}$ by replacing one of the existing unused (or seldom-used) internal filters with an $8 \mathrm{MHz}$ filter and

\footnotetext{
${ }^{1}$ The VLBA record normally operates with a single headstack with a maximum formatted-data-rate of 256 Mbits/sec. However, the transport has positions for up to four headstacks, three of which are vacant for a standard VLBA recorder. For the $1 \mathrm{Gbit} / \mathrm{sec}$ demonstration, a standard VLBA tape recorder was outfitted with four standard headstacks to occupy all headstack-positions available on the Honeywell 96 transport. A standard VLBA formatter was used to drive the 36 tracks on each headstack at a rate of $9 \mathrm{Mbits} / \mathrm{sec} / \mathrm{track}$ for a total of $1.296 \mathrm{Gbits} / \mathrm{sec}$ total data rate. With the normal formatting overhead of $9 / 8$, this corresponds to a 1.152 Gbits/sec data rate for formatted VLBI data. Data were recorded for several adjacent passes on the tape to simulate a portion of an actual experiment. For compatibility with the Mark IIIA correlator, data were recorded in Mark IIIA format at 33,000 bits/inch /track.
}

Following recording of the data, the Mark IIIA correlator at Haystack was used to auto-correlate the recorded tape (in several passes) to verify the quality and validity of the data. All data were read properly and the processing through the correlator produced nominal results. 
changing the gain-compensation resistors.

- $\quad$ Replace existing Mark IIIA formatter with new Mark IV formatter:

- Plug-in replacement for existing formatter

- 16 channels at (up to) 32 Msamples/sec

- 1 or 2 bits/sample

- channel-to-head cross-point matrix switch

- $\quad$ support of many VLBA-compatibility modes

- expandable to 32 channels at $32 \mathrm{Msamples} / \mathrm{sec}$ (2048 Mbits/sec total)

- Replace existing write interfaces with VLBA-style read/write interfaces which use the same heads for read and write

- Use both existing headstacks for both reading and writing, for a total of 64 available tracks

- $\quad$ Add necessary write electronics to support 64 heads

A complete Mark IV data-acquisition system has the following characteristics:

- 64 tracks at $16 \mathrm{Mbits} / \mathrm{sec} /$ track for a total of $\mathbf{1 0 2 4} \mathrm{Mbits} / \mathrm{sec}$ formatted data

- Uses the same 16 micron-thick tape chosen by the VLBA:

- $\quad 18000^{\prime}$ of tape on standard 14" reel

- longitudinal density: $56,000 \mathrm{bits} / \mathrm{inch} / \mathrm{track}$

- $\quad$ tape speed: $320 \mathrm{ips}$ (16 Mbits/sec/track)

- $\quad$ record time per pass: 11.25 minutes

- $\quad$ record time per tape (6 passes): 67.5 minutes

- $\quad$ 95\% SNR improvement over present maximum-data-rate Mark IIIA

- Full backward compatibility to all standard Mark IIIA operating modes, as well as compatibility with as many VLBA modes as possible

\subsection{Compatibility Between Various Recording Systems}

The subject of compatibility between various VLBI data-acquisition system and correlators is a very complex one. In this section we will only attempt to summarize the salient similarities and differences of the various systems; a complete discussion is beyond the scope of this paper. The major differences between the Mark IIIA, Mark IV, and VLBA system can be summarized as follows:

- the Mark IIIA has 14 video converters, each with a maximum bandwidth of 4 $\mathrm{MHz} /$ sideband, supports only $1 \mathrm{bit} / \mathrm{sample}$, data-replacement format only, and maps data from one video-converter sideband to one tape-track (i.e. no data-multiplexing or 'barrel-rolling'), with a maximum data rate of $224 \mathrm{Mbits} / \mathrm{sec}$ on 28 tracks

- the Mark IV has 16 video converters, each with a maximum bandwidth of 8 
$\mathrm{MHz} /$ sideband, supports 1 or 2 bits/sample, data-replacement format only, support several modes of multiplexing, with a maximum data rate of $1024 \mathrm{Mbits} / \mathrm{sec}$ on 64 tracks

- a single standard VLBA data-acquisition system has 8 video converters, each with a maximum bandwidth of $16 \mathrm{MHz} /$ sideband, supports 1 or 2 bits/sample, data-replacement or non-data-replacement format, many mode of multiplexing and barrel-rolling, with a maximum data rate of $256 \mathrm{Mbits} / \mathrm{sec}$ on 32 tracks; each VLBA station will (eventually) be equipped with 2 standard VLBA data-acquisition systems; on special order, a VLBA dataacquisition-system may be equipped with 14 video converters

Table I shows a comparison of the data-recording capabilities of the Mark IIIA, the Mark IV, and 'standard' and 'augmented' VLBA data-acquisition systems. The major differences to note are numbers of channels and channel bandwidths. As you can see from an examination of Table I, there are many compatible modes between Mark IV and VLBA, though the reader is referred to official VLBA documentation for a complete discussion of the capabilities of the VLBA data-acquisition-system.

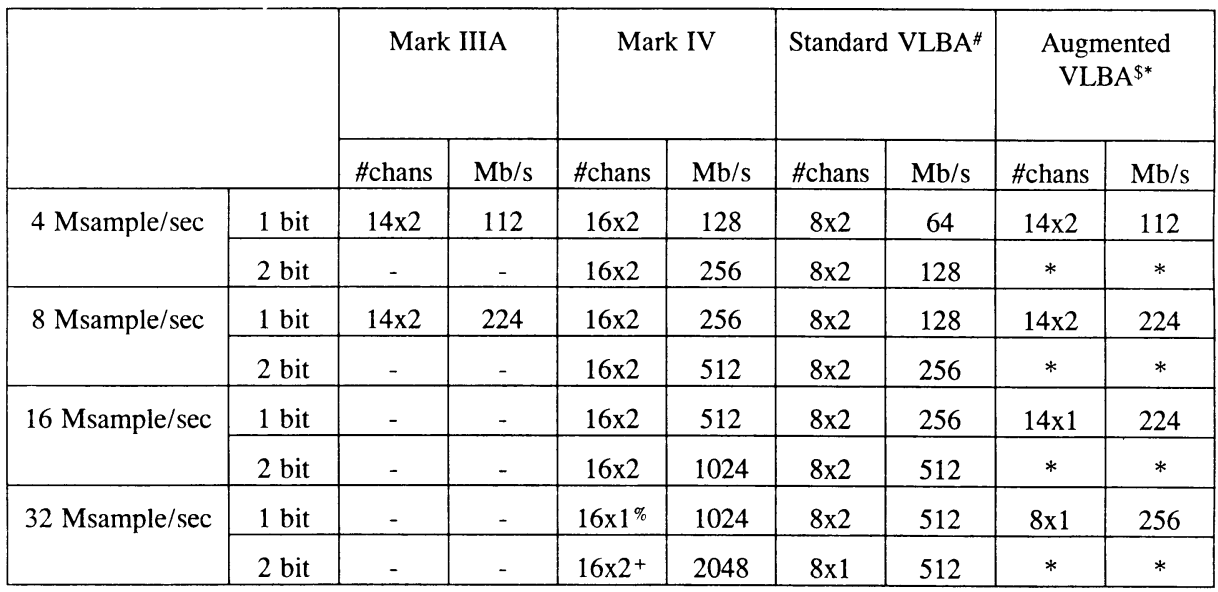

Table I: Data-Acquisition-System Capability/Compatibility

\footnotetext{
\# 8 video converters, 2 formatters, 2 recorders

$\$ 14$ video converters, 1 formatter, 1 recorder

* Augmented VLBA supports all Standard VLBA modes up to $256 \mathrm{Mb} / \mathrm{s}$ by moving two cables

$\%$ Requires augmenting Mark III video converters to $16 \mathrm{MHz}$ channel bandwidth

+ Augmented with 2 additional headstacks, additional board in formatter, 16-MHz video-converter bandwidth
} 


\section{Mark IV Correlator}

The Mark IV data-recording system creates data in basically the same Mark IIIA tape format that has been supported by correlators for some years. In fact, the existing Haystack correlator (designated 'Mark IIIA', but not to be confused with the Mark IIIA recording system) or the VLBA correlator could process the data from the Mark IV recording system, but would require several passes through the tape. This is clearly impractical given the established observation rates of both astronomers and geodesists. Therefore, we consider here a correlator design that can properly complement the Mark IV recording system.

Based on the requirements for processing data from the Mark IV recording system, a new Mark IV correlator system is being constructed. The performance goals for this system are:

- $1-\mathrm{Gbit} / \mathrm{sec} / \mathrm{station}$ processing speed (16 channels at $32 \mathrm{Msamples} / \mathrm{sec} / \mathrm{channel})$

- Expandable to 32 stations

- Compatible with Mark IIIA, Mark IV, and VLBA data-acquisition systems

A study was initiated to chose the best architecture for the new correlator. Several different architectures, including both X-F and F-X designs were considered. Upon review of these studies, the following decisions were made:

- A station-based architecture was chosen to minimize the amount of hardware necessary in a large correlator system. This architecture implies that the data from the VLBI dataplayback system is reassembled and pre-delayed in a station unit prior to being transmitted to the correlation unit.

- The correlation algorithms will follow the X-F algorithms designed and used successfully on the Mark III and Mark IIIA correlators. These algorithms have been welldeveloped and are well understood. Furthermore, it is relatively straightforward to design the system to make extensive use of the tradeoff between number-of-lags and number-ofchannels, so that the flexibility of the correlator is significantly enhanced for many applications.

- A new full-custom VLSI correlator chip is being developed to significantly enhance the power of the correlator unit and reduce the amount of hardware in a large correlator system.

- Adopt the same basic playback machine design developed by Haystack for the VLBA. This playback machine will support tape-playback speeds to 320 ips, compared with the existing 135 ips of the current playback machines.

- Improved modeling capabilities to reduce possibilities of systematic errors

- Operation at data rates up to $32 \mathrm{Mbits} / \mathrm{sec}$

- Compatibility with Mark IIIA, Mark IV, and some VLBA-format modes

- Significantly-improved computer support utilizing the Unix operating system on a modern platform 
- Full real-time capability integrated into the design in anticipation of real-time VLBI processing in the future

- Improved hardware reliability and ease of maintenance

\subsection{Station Unit}

The primary function of the station unit is to reconstruct playback-data from a single station and pre-delay it according to a model provided by the host computer. Because the Mark IV and VLBA systems are capable of multiplexing data from a single channel onto several tracks, this multiplexing process must be reversed by the station unit in order to reconstruct a single channel of data and properly condition it for transmission to the correlator unit. This is done in four steps:

1. The clock is recovered from each of (up to) 64 tracks of data in a 'clock-recovery' module.

2. The clock-recovered data is passed to a 'track-recovery' module, which detects embedded sync words, validates CRC, and counts parity errors.

3. A 'de-formatter' module analyzes frames of data recovered from individual tracks and places them in the proper order to properly reconstruct the original 'channel' data streams. This process undoes any multiplexing or barrel-rolling applied during datataking operations.

4. Each 'channel' data-stream is delayed (relative to the time written on the tape) according to a polynomial model provided by the correlator control computer, and clocked out of the station unit at a fixed clock rate (up to $32 \mathrm{MHz}$ ). Individual data samples are deleted or added as necessary to keep the 'delay error' within +-0.5 samples of the polynomial mode.

\subsection{Correlator Module and VLSI Correlator Chip}

A new correlator module with a new full-custom VLSI chip will form the heart of the Mark IV correlator. The overall processing capability of the Mark IV module is expected to exceed the present Haystack correlator module by more than a factor of 100 , where processing capability is defined as (max-bit-rate)x(number lags)/unit-cost. The module will be mated to a standard VME-based backplane, which provides a standardized I/O system with high-speed data capability, and the ability to use standardized low-cost in-crate processors for real-time control. An on-board microprocessor will provide the power for intelligent decision-making and real-time computational tasks.

The new VLSI correlator chip being developed for Mark IV will have the following characteristics:

- Full-custom CMOS with 1,000,000 transistors

- 1-bit or 2-bit samples

- Each chip will have 512 lags which can be rearranged internally into several independent correlator sections, each having from 16 to 512 lags, in both real and complex configurations. 
- $64 \mathrm{MHz}$ minimum clock rate

- internal 32-bit quadrature phase generators and rotator for rotation at (up to) full bandwidth

- $\quad$ internal +/-90 deg bit-shift/phase-shift algorithm

- internal vernier delay generators to properly track delay errors

- 24-bit latchable ripple counters on each lag

The development cost of this chip is being shared by NASA (also representing USNO and NOAA) and by the Smithsonian Center for Astrophysics, who plan to use the same chip in the Smithsonian sub-millimeter array being constructed on Mauna Kea, Hawaii. The chip is being designed and layed-out by the NASA Space Engineering Research Center for VLSI System Design, under contract to Haystack Observatory. The first prototypes samples of this chip are expected in late 1993.

The correlation unit is being developed by Haystack Observatory with cooperative design assistance from Dr. Albert Bos of NFRA, Netherlands and Dr. Brian Anderson of Jodrell Bank, and is expected to be used in a new large correlator being funded by the European VLBI Network (EVN) as well as in the Mark IV correlator. Each correlator board (=correlator unit) will contain 32 VLSI correlator chips and be capable of processing a single 32 Msample/sec channel from up to 120 baselines (16 stations), as well as many combinations of fewer stations and more lags/baseline. Only 16 of these correlator boards will be required to support full continuum processing of $1 \mathrm{Gbit} /$ station data from 16 stations.

\section{Summary}

The Mark IV VLBI system is being developed as the next logical step in the evolution of VLBI data-acquisition systems. With a relatively low-cost upgrade to existing Mark IIIA systems, the Mark IV economically doubles the sensitivity by pushing recorded data rates to $1 \mathrm{Gbit} / \mathrm{sec}$, with expansion capability to $2 \mathrm{Gbit} / \mathrm{sec}$.

The Mark IV development program has received support from a large number of institutions, including NASA, U.S. Naval Observatory, National Oceanographic and Atmospheric Administration, and NRAO in the U.S. The Mark IV correlator development is being supported by NASA and USNO, with contributions and collaborative efforts from Smithsonian Astrophysical Observatory, Netherlands Foundation for Research in Astronomy, Nuffield Radio Astronomy Laboratories (Jodrell Bank), and the European VLBI Network. 